May 2013

"Atkinson-Stiglitz and Ramsey reconciled: Pareto efficient taxation and pricing under a break-even constraint"

Helmuth Cremer and Firouz Gahvari 


\title{
Atkinson-Stiglitz and Ramsey reconciled: Pareto efficient taxation and pricing under a break-even constraint*
}

\author{
Helmuth Cremer \\ Toulouse School of Economics \\ (University of Toulouse and Institut universitaire de France) \\ Toulouse, France \\ Firouz Gahvari \\ Department of Economics \\ University of Illinois at Urbana-Champaign \\ Urbana, IL 61801, USA
}

May 2012, revised May 2013

*This paper has been presented in seminars at CESifo, Uppsala University, the University of Cologne and University Nova (Lisbon). We thank all the participants and in particular Felix Biebrauer, Soren Blomquist, Denis Epple, Kenneth Kletzer and Catarina Reis for their helpful suggestions and comments. We also thank Jean-Tirole for a number of inspiring discussions about the underlying problem. 


\begin{abstract}
The Ramsey tax problem examines the design of linear commodity taxes to collect a given tax revenue. This approach has been seriously challenged by Atkinson and Stiglitz (1976) who show that (under some conditions) an optimal income tax makes commodity taxes redundant. Accordingly, the Ramsey approach to taxation is considered as dated.

In the meantime, the Ramsey setting has had a second life as model of regulatory pricing. Boiteux (1956) studies linear pricing of a regulated multi-product monopoly that has to cover some "fixed cost" through markups on the different products (equivalent to taxes). While the scope of regulation has declined, Ramsey-Boiteux pricing continues to play an important role.

This paper examines if the optimal tax and regulatory pricing approaches to Ramsey pricing can be reconciled. We incorporate the two objectives of revenue raising for financing the government's expenditures and a regulated firm's fixed cost into a single framework.

The first major lesson of our study is that the existence of a break-even constraint not only requires taxation of goods produced by the public/regulated firm but also the taxation of other goods. Next, we consider two special cases for which the Ramsey model yields simple and well-known results; namely, the cases of independent Hicksian and Marshallian demand curves. In both the Ramsey solution implies the so-called inverse elasticity rule.

In the separable Hicksian demand case, the private goods (not included in the breakeven constraint) continue to go untaxed as in the Atkinson and Stiglitz setting. In the case where Marshallian demands are independent, the effect of the break-even constraint spills over to the other goods who no longer go untaxed. We continue to get inverse elasticity rules; however, their structure differs from the traditional expressions. In particular, there is no covariance (or similar) term that captures redistributive considerations. Instead, they contain "tax revenue terms" that measure the social value of the extra tax revenues generated from demand variations that follow the (compensating) adjustments in disposable income.

Finally, we study the most celebrated general result obtained in the Ramsey model; namely, the (un)equal proportional reduction in compensated demands property. We find that the redistributive considerations are once again replaced by tax revenue terms.
\end{abstract}




\section{Introduction}

The Ramsey tax problem is one of the cornerstones of optimal tax theory. The question it examines is that of designing linear (proportional) commodity taxes to collect a given tax revenue. Labor income is not taxed at all or subject to a linear tax. ${ }^{1}$ The first analysis of this problem is due to Ramsey (1927) but most of the formal results were derived in the 1970s starting with the seminal paper by Diamond and Mirrlees (1971). This was followed by literally hundreds of papers. Baumol and Bradford (1970) and Sandmo (1976) provide an interesting history of this subject. The main point that this literature makes is that, except in very special cases, commodity taxes should not be uniform and that they should be set to balance efficiency and equity considerations. The efficiency aspects appear most obviously in the inverse elasticity rules derived when demand functions are separable (Hicksian or Marshallian). These efficiency driven tax rules which are regressive because goods with low price elasticities are often necessities consumed proportionately more by poorer households. This redistributive bias is mitigated by the equity terms in the tax rules (often covariance terms). These tend to increase the tax rate on goods that are consumed proportionately more by richer individuals.

This approach to tax design has been seriously challenged by Atkinson and Stiglitz (1976) who show that under some conditions an optimal income tax is sufficient to implement any incentive compatible Pareto-efficient allocation. In other words, commodity taxes are not needed (or should be uniform), and the Ramsey results are merely an artifact of the restriction that income taxes must be linear (an ad hoc and inconsistent assumption given the assumed information structure). The Atkinson and Stiglitz (AS) result has far reaching implications for the design of optimal tax systems. In particular,

\footnotetext{
${ }^{1}$ In the original Ramsey problem, individuals are alike and there is no income tax. With heterogeneous individuals, one also allows for a uniform lump-sum tax or rebate (and possibly a linear tax on labor income).
} 
whenever it applies, it implies that in-kind transfers are not useful and prices should not be used for redistribution (even in a second best setting).

It is by now well understood though that the AS result also has its own limitations. In particular, it may not hold under uncertainty (Cremer and Gahvari, 1995) or under multi-dimensional heterogeneity (Cremer, Gahvari and Ladoux, 1998, and Cremer, Pestieau and Rochet, 2001) and redistribution through prices may then once again be second-best optimal (Cremer and Gahvari, 1998 and 2002). Still, these limitations notwithstanding, it is fair to say that the Ramsey approach to taxation is considered as dated and no longer "state of the art". It does continue to occupy a prominent place in all advanced textbooks, but it is taught mainly as an introduction to tax design and not because of its practical relevance.

In the meantime, the Ramsey setting has had a more or less independent secondor some might argue first - life as model of regulatory pricing. In his pioneering paper, Boiteux (1956) studies linear pricing of a regulated multi-product monopoly that has to cover some "fixed cost" (for instance the infrastructure cost of the network). This is to be achieved through markups on the monopoly's different products (equivalent to taxes). ${ }^{2}$ Formally, this problem is equivalent to a Ramsey tax model with the fixed cost playing the role of the government's tax revenue requirement. While the scope of regulation has declined over the last decades, Ramsey-Boiteux (RB) pricing continues to play an important role in the sectors still subject to some form of regulation. A prominent example is the postal sector in the US where Ramsey-Boiteux pricing remains an important benchmark in regulatory hearings; see Crew and Kleindorfer (2012). As a matter of fact, not only has RB pricing kept its position but it has even found new applications in setting of regulatory reform and market liberalization. For instance, while the original Boiteux model concerns a monopoly, Ware and Winter (1985) show that generalized RB rules prevail in imperfectly competitive markets. Furthermore,

\footnotetext{
${ }^{2}$ We follow the terminology used in the regulation literature but, in reality, this is a quasi-fixed cost relevant also in the long-run (a non convexity in the production set).
} 
Laffont and Tirole (1990) argue that the network access an incumbent operator has to provide to its upstream competitors should be priced on the basis of RB logic. Another interesting result, shown by Vogelsang and Finsinger (1979), is that Ramsey prices can be decentralized through an iterative procedure based on a global price cap; see also Laffont and Tirole (2000, page 67). Finally, the idea that prices ought to be used for redistributive purposes is the rational for a great deal of regulatory policies including social tariffs and more generally universal service requirements; see Cremer et al. (2001) for a discussion of the theoretical foundations of these policies and their practical implementation. ${ }^{3}$

To sum up, optimal tax and regulatory pricing literatures appear to have diverged in the way they view the implications of the AS result. This paper examines if the two approaches can be reconciled. To this end, we incorporate the two objectives of revenue raising for financing the government's expenditures and a regulated firm's fixed cost into a single framework. While the two decisions are formally equivalent when each is addressed separately, a comprehensive analysis requires a unified framework. Our setting is that of AS. More precisely, we consider a mixed taxation setting à la Christiansen (1984) that combines nonlinear income taxation with linear pricing (taxation) of consumption goods. We assume that a subset of the goods are produced by a public or regulated firm that has to cover a fixed cost through markups on the different commodities it sells. ${ }^{4}$ This constraint gives rise to a "break-even" constraint on the part of the firm. This comes on top of the overall government's budget constraint. The question is if this break-even constraint revives any of the Ramsey pricing or taxation features (for goods produced by the public/regulated firm as well as for other goods).

\footnotetext{
${ }^{3}$ While it is true that regulators and especially competition authorities are often reluctant to accept Ramsey pricing arguments, this is not because of the AS result. Their objection is more of legal and procedural nature. In particular, Ramsey prices are often viewed as "discriminatory" and subject to informational problems when the operator is better informed about demand condition than the regulator.

${ }^{4}$ Whether this firm is public, as in Boiteux's world, or private but regulated does not matter. Either way, one implicitly assumes that the firms' revenues must also cover some "fair" rate of return on capital.
} 
We start by formulating the general problem a government faces when determining incentive compatible Pareto-efficient allocations in a discrete economy wherein individuals differ in their productivity. ${ }^{5}$ Individual types and labor supplies are not publicly observable, but pre-tax incomes are. This rules out type-specific lump-sum taxes but allows for nonlinear income taxation. Individual consumption levels of goods, whether subject to regulation or not, are not observable so that nonlinear taxation and/or pricing of goods are not possible. However, anonymous transactions of goods making linear commodity taxation feasible. This information structure is by now standard in the taxation literature. ${ }^{6}$ The key feature of our setup is that some of the goods are produced by a public/regulated firm subject to a break-even constraint.

While we optimize over all tax instruments including income, we are not concerned with the properties of the income tax schedule. Our aim is to study the commodity taxation and pricing rules that emerge for goods that are produced by public/regulated firms as well as those produced subject to no regulation. We derive these rules for general preferences but concentrate on to the case of weakly-separable preferences between labor supply and goods that underlies the AS result. In this way, we demonstrate the first major lesson of our study; namely, that the existence of a break-even constraint not only requires taxation of goods produced by the public/regulated firm but also the taxation of other goods. In other words, taxation of privately-produced goods are generally needed to offset the distortions created by the public/regulated firm's departure from marginal cost pricing. We then illustrate and elaborate on these lessons by studying a simple framework with one publicly-provided and two privately-provided goods.

Next, we recast and derive the Ramsey rules for setting wherein taxes (markups) are used to finance both a revenue requirement and a fixed cost (rather than only one of the two as in the traditional model). While this yields predictable results and is not of much interest in itself, it is a useful reference point. It also encapsulates and highlight

\footnotetext{
${ }^{5}$ Our results will not change if a continuous distribution of types are considered.

${ }^{6}$ This amounts to adding a nonlinear income tax to the Ramsey tax/pricing problem.
} 
the effects of the break-even constraint per se. Then, returning to our setting, to gain a better intuition into the nature of the tax/pricing rules, we consider two special cases for which the Ramsey model yields simple and well-known results; namely, the cases of independent Hicksian and Marshallian demand curves. In both the Ramsey solution implies the so-called inverse elasticity rule.

In the separable Hicksian demand case, we find that the private goods (not included in the break-even constraint) continue to go untaxed as in the Atkinson and Stiglitz setting. On the other hand, the pricing rules used by the public firm are purely efficiencydriven Ramsey rules. Goods are taxed inversely to their compensated demand elasticity regardless of their distributional implications. Redistribution is taken care of by the income tax allowing the public firm's prices to be adjusted for revenue raising (as in the Ramsey model with identical individuals).

Results become less predictable in the case where Marshallian demands are independent. Then the effect of the break-even constraint is no longer confined to the goods subject to this constraint. Instead, it spills over to the other goods who no longer go untaxed. We continue to get inverse elasticity rules as in the Ramsey model; however, their structure differs from the traditional expressions one gets in the Ramsey model without a break-even constraint. On the one hand, they are more complicated than the pure efficiency rules. On the other hand, there is no covariance (or similar) term that captures redistributive considerations. Instead, they contain "tax revenue terms" that measure the social value of the extra tax revenues generated from demand variations that follow the (compensating) adjustments in disposable income. These terms lead to predictions that are similar to those coming from redistributive terms in the many-household Ramsey model without a break-even constraint; namely, that goods with higher demand elasticities should be taxed more heavily (see below).

Finally, we study what is arguably the most celebrated general result obtained in the Ramsey model; namely, the (un)equal proportional reduction in compensated de- 
mands property. We show that, in contrast to the single-household Ramsey model, the reductions differ across goods. This in and of itself is not particularly surprising given the presence of heterogeneous households. More interestingly, when compared to the many-household Ramsey case without the break-even constraint, we find that the redistributive considerations are once again replaced by tax revenue terms.

\section{The model}

There are $N$ types of individuals, indexed $j=1, \ldots, N$, who differ in their wages, $w^{j}$, but have identical preferences. All goods are produced at a constant marginal cost which we normalize to one. However, some of the goods are produced by a public or regulated firm which incurs a fixed cost. The firm is constrained to break even by marking up its marginal costs. ${ }^{7}$ Denote the goods that private sector produces by $\underline{x}=\left(x_{1}, x_{2}, \ldots, x_{n}\right)$ and goods that the public firm produce by $\underline{y}=\left(y_{1}, y_{2}, \ldots, y_{m}\right)$. Let $\underline{p}=\left(p_{1}, p_{2}, \ldots, p_{n}\right)$ denote the consumer price of $\underline{x}$ and $\underline{q}=\left(q_{1}, q_{2}, \ldots, q_{n}\right)$ the consumer prices of $\underline{y}$. Finally, denote the commodity tax rates on $\underline{x}$ by $\underline{t}=\left(t_{1}, t_{2}, \ldots, t_{n}\right)$ and the public firms' commodity-tax-cum-mark-ups by $\underline{\tau}=\left(\tau_{1}, \tau_{2}, \ldots, \tau_{m}\right)$. We have $p_{i}=1+t_{i}$ $(i=1,2, \ldots, n)$ and $q_{s}=1+\tau_{s}(s=1,2, \ldots, m)$.

Individual consumption levels are not publicly observable but anonymous transactions can be observed. Consequently, commodity taxes must be proportional and public sector prices are linear. For the remaining variables, the information structure is the one typically considered in mixed taxation models; see e.g., Christiansen (1984) and Cremer and Gahvari (1997). In particular, an individual's type, $w^{j}$, and labor input, $L^{j}$, are not publicly observable; his before-tax income, $I^{j}=w^{j} L^{j}$, on the other hand, is. Consequently, type-specific lump-sum taxation is ruled out but non-linear taxation of incomes is feasible.

\footnotetext{
${ }^{7}$ Alternatively one can think of a privately owned regulated firm whose prices are set to cover cost plus a fair rate of return on capital.
} 
To characterize the (constrained) Pareto-efficient allocations we derive an optimal revelation mechanism. For our purpose, a mechanism consists of a set of type-specific before-tax incomes, $I^{j}$ 's, aggregate expenditures on private goods, $c^{j}$ 's, a vector of consumer prices (same for everyone) for $\underline{x}$ and for $\underline{y}, \underline{p}$ and $\underline{q}$. To proceed further, it is necessary to consider the optimization problem of an individual for a given mechanism $(\underline{p}, \underline{q}, c, I)$. Formally, given any vector $(\underline{p}, \underline{q}, c, I)$, an individual of type $j$ maximizes utility $u=u\left(\underline{x}, \underline{y}, I / w^{j}\right)$ subject to the budget constraint $\sum_{i} p_{i} x_{i}+\sum_{s} q_{s} y_{s}=c$.

The resulting demand functions for $\underline{x}$ and $\underline{y}$ are denoted by $x_{i}=x_{i}\left(\underline{p}, \underline{q}, c, I / w^{j}\right)$ and $y_{s}=y_{s}\left(\underline{p}, \underline{q}, c, I / w^{j}\right)$. Substituting in the utility function yields the indirect utility function

$$
v\left(\underline{p}, \underline{q}, c, I / w^{j}\right) \equiv u\left[\underline{x}\left(\underline{p}, \underline{q}, c, I / w^{j}\right), \underline{y}\left(\underline{p}, \underline{q}, c, I / w^{j}\right), I / w^{j}\right] .
$$

Thus, a $j$-type individual who is assigned $c^{j}, I^{j}$ will have demand functions and an indirect utility function given by

$$
\begin{aligned}
x_{i}^{j} & =x_{i}\left(\underline{p}, \underline{q}, c^{j}, I^{j} / w^{j}\right), \\
y_{s}^{j} & =y_{s}\left(\underline{p}, \underline{q}, c^{j}, I^{j} / w^{j}\right), \\
v^{j} & =v\left(\underline{p}, \underline{q}, c^{j}, I^{j} / w^{j}\right) .
\end{aligned}
$$

Similarly, the demand functions and the indirect utility function for a $j$-type who claims to be of type $k$, the so-called mimicker, is given by

$$
\begin{aligned}
x_{i}^{j k} & =x_{i}\left(\underline{p}, \underline{q}, c^{k}, I^{k} / w^{j}\right), \\
y_{s}^{j k} & =y_{s}\left(\underline{p}, \underline{q}, c^{k}, I^{k} / w^{j}\right), \\
v^{j k} & =v\left(\underline{p}, \underline{q}, c^{k}, I^{k} / w^{j}\right) .
\end{aligned}
$$




\subsection{Pareto-efficient (constrained) allocations}

Denote the government's external revenue requirement by $\bar{R}$ and the fixed costs of public firms by $F$. Constrained Pareto-efficient allocations are described, indirectly, as follows. ${ }^{8}$ Maximize

$$
\sum_{j=1}^{H} \eta^{j} v\left(\underline{p}, \underline{q}, c^{j}, I^{j} / w^{j}\right)
$$

with respect to $p_{2}, p_{3}, \ldots, p_{n}, \underline{q}, c^{j}$ and $I^{j}$ where $\eta^{j}$ s are constants with the normalization $\sum_{j=1}^{H} \eta^{j}=1$. The maximization is subject to the resource constraint

$$
\sum_{j=1}^{H} \pi^{j}\left[\left(I^{j}-c^{j}\right)+\sum_{i=1}^{n}\left(p_{i}-1\right) x_{i}^{j}+\sum_{s=1}^{m}\left(q_{s}-1\right) y_{s}^{j}\right] \geq \bar{R}+F,
$$

the break-even constraint

$$
\sum_{j=1}^{H} \pi^{j}\left[\sum_{s=1}^{m}\left(q_{s}-1\right) y_{s}^{j}\right] \geq F
$$

and the self-selection constraints

$$
v^{j} \geq v^{j k}, \quad j, k=1,2, \ldots, H
$$

Denote the Lagrangian expression by $\mathcal{L}$, and the Lagrangian multipliers associated with the resource constraint (8) by $\mu$, the public firms' break-even constraint (9) by $\delta$, and with the self-selection constraints (10) by $\lambda^{j k}$. We have ${ }^{9}$

$$
\begin{aligned}
\mathcal{L}= & \sum_{j} \eta^{j} v^{j}+\mu\left\{\sum_{j} \pi^{j}\left[\left(I^{j}-c^{j}\right)+\sum_{i=1}^{n}\left(p_{i}-1\right) x_{i}^{j}+\sum_{s=1}^{m}\left(q_{s}-1\right) y_{s}^{j}\right]-\bar{R}-F\right\} \\
& +\delta\left\{\sum_{j} \pi^{j}\left[\sum_{s=1}^{m}\left(q_{s}-1\right) y_{s}^{j}\right]-F\right\}+\sum_{j} \sum_{k \neq j} \lambda^{j k}\left(v^{j}-v^{j k}\right) .
\end{aligned}
$$

\footnotetext{
${ }^{8}$ Indirectly because the optimization is over a mix of quantities and prices. Then, given the commodity prices, utility maximizing individuals would choose the quantities themselves.

${ }^{9}$ Recall that $p_{1}=1$ so that

$$
\sum_{i=1}^{n}\left(p_{i}-1\right) x_{i}^{j}=\sum_{i=2}^{n}\left(p_{i}-1\right) x_{i}^{j}
$$
}


The first-order conditions of this problem with respect to $I^{j}, c^{j}$, for $j, k=1,2, \ldots, H$, and $p_{i}, q_{s}$, for $i=2,3, \ldots, n$, and $s=1,2, \ldots, m$, characterize the Pareto-efficient allocations constrained both by the public firms' break-even constraint, the resource constraint, the self-selection constraints, as well as the linearity of commodity tax rates. They are derived in the Appendix.

One feature of this setup is of note. With $\underline{x}$ and $\underline{y}$ being homogeneous of degree zero in $p, q$, and $c$, consumer prices can determined only up to a proportionality factor. Consequently, we do not optimize over $p_{1}$ and set its value at $p_{1}=1$. In the absence of the break-even constraint, this normalization is without any loss of generality. With a binding break-even constraint, it does involve a restriction, namely that we rule out an across the board uniform increase in all (consumer) prices to ensure that the public firms revenues cover fixed costs. While this is a theoretical possibility in this setting, it does not appear to be a realistic course of action. As a matter of fact such a policy would be in contradiction with the very idea of imposing a break-even constraint in the first place. ${ }^{10}$

\section{Atkinson and Stiglitz theorem and optimal commodity taxes}

In the standard mixed taxation model without the break-even constraint, assuming preferences are weakly separable in goods and labor supply, the Atkinson and Stiglitz (1976) theorem on the redundancy of commodity taxes holds. The particular feature of separability that drives the AS result is the property that a $j$-type who pretends to be

\footnotetext{
${ }^{10} \mathrm{In}$ a setting where tax policy is restricted by informational considerations only, the break-even constraint could be undone by a simple lump-sum transfers (from the government to the operator). The $\mathrm{RB}$ approach rules out such a transfer in an ad hoc way. This reflects some considerations which are not explicitly addressed in the models. For instance, for political economy reasons it is required that natural gas users (and not the taxpayer) are responsible to finance the transportation network of pipeline and pumping stations.
} 
of type $k$ will have the same demand as type $k$. That is,

$$
\begin{aligned}
& x_{i}^{j k}=x_{i}^{k}=x_{i}\left(\underline{p}, \underline{q}, c^{k}\right), \\
& y_{s}^{j k}=y_{s}^{k}=y_{s}\left(\underline{p}, \underline{q}, c^{k}\right) .
\end{aligned}
$$

This arises because with separability preferences take the following form $u=u\left(f(\underline{x}, \underline{y}), I / w^{j}\right)$. Under this circumstance, the (conditional) demand functions for $\underline{x}$ and $\underline{y}$ specified in equations (1)-(2) and (4)-(5) will be independent of $I / w^{j}$ so that $x_{i}=x_{i}(\underline{p}, \underline{q}, c)$ and $y_{s}=y_{s}(\underline{p}, \underline{q}, c)$. Moreover, the indirect utility function too will be weakly separable in $(\underline{p}, \underline{q}, c)$ and $I / w^{j}$ and written as $v\left(\phi(\underline{p}, \underline{q}, c), I / w^{j}\right)$.

The above property also has far reaching implications for optimal commodity taxes; both those produced by the public firm as well as privately. To derive these, introduce the compensated version of demand functions (1)-(2). Specifically, denote the compensated demand for a good by a "tilde" over the corresponding variable. Let $\Delta$ denote the $(n+m-1) \times(n+m-1)$ matrix derived from the Slutsky matrix, aggregated over all individuals, by deleting its first row and column,

$$
\Delta=\left(\begin{array}{cccccc}
\sum_{j} \pi^{j} \frac{\partial \tilde{x}_{2}^{j}}{\partial p_{2}} & \cdots & \sum_{j} \pi^{j} \frac{\partial \tilde{x}_{2}^{j}}{\partial p_{n}} & \sum_{j} \pi^{j} \frac{\partial \tilde{x}_{2}^{j}}{\partial q_{1}} & \cdots & \sum_{j} \pi^{j} \frac{\partial \tilde{x}_{2}^{j}}{\partial q_{m}} \\
\vdots & \ddots & \vdots & \vdots & \ddots & \vdots \\
\sum_{j} \pi^{j} \frac{\partial \tilde{x}_{n}^{j}}{\partial p_{2}} & \cdots & \sum_{j} \pi^{j} \frac{\partial \tilde{x}_{n}^{j}}{\partial p_{n}} & \sum_{j} \pi^{j} \frac{\partial \tilde{x}_{n}^{j}}{\partial q_{1}} & \cdots & \sum_{j} \pi^{j} \frac{\partial \tilde{x}_{n}^{j}}{\partial q_{m}} \\
\sum_{j} \pi^{j} \frac{\partial \tilde{y}_{1}^{j}}{\partial p_{2}} & \cdots & \sum_{j} \pi^{j} \frac{\partial \tilde{y}_{1}^{j}}{\partial p_{n}} & \sum_{j} \pi^{j} \frac{\partial \tilde{y}_{1}^{j}}{\partial q_{1}} & \cdots & \sum_{j} \pi^{j} \frac{\partial \tilde{y}_{1}^{j}}{\partial q_{m}} \\
\vdots & \ddots & \vdots & \vdots & \ddots & \vdots \\
\sum_{j} \pi^{j} \frac{\partial \tilde{y}_{m}^{j}}{\partial p_{2}} & \cdots & \sum_{j} \pi^{j} \frac{\partial \tilde{y}_{m}^{j}}{\partial p_{n}} & \sum_{j} \pi^{j} \frac{\partial \tilde{y}_{m}^{j}}{\partial q_{1}} & \cdots & \sum_{j} \pi^{j} \frac{\partial \tilde{y}_{m}^{j}}{\partial q_{m}}
\end{array}\right) .
$$

We prove in the Appendix that in this case optimal commodity taxes satisfy the following equations,

$$
\left(\begin{array}{c}
t_{2} \\
\vdots \\
t_{n} \\
\left(1+\frac{\delta}{\mu}\right) \tau_{1} \\
\vdots \\
\left(1+\frac{\delta}{\mu}\right) \tau_{m}
\end{array}\right)=-\frac{\delta}{\mu} \Delta^{-1}\left(\begin{array}{c}
0 \\
\vdots \\
0 \\
\sum_{j} \pi^{j} y_{1}^{j} \\
\vdots \\
\sum_{j} \pi^{j} y_{m}^{j}
\end{array}\right)
$$


Observe that if $F=0$, i.e. if there is no fixed cost, the break-even constraint is irrelevant. Under this circumstance, $\delta=0$ and the right-hand side of (13) consists of a vector of $n-1+m$ zeros. One then obtains $t_{i}=0$ and $\tau_{s}=0$ (marginal cost pricing) for all $i=1, \ldots, n$ and $s=1, \ldots, m$, and returns to the Atkinson and Stiglitz result that commodity taxes are redundant. With $F>0$, the break-even constraint is necessarily violated under marginal cost pricing so that $\delta>0$. In this case, the first $(n-1)$ lines on the of (13) continue to be zero, but the other lines differ from zero. It then follows that the solution no longer implies all $t$ 's and all $\tau$ 's are zero. Nor will it be the case that the $t$ 's are necessarily zero.

This latter point, that the existence of a break-even point requires not only the taxation of goods produced by the public firm but also the taxation of privately-provided goods, constitutes the first major lesson of our study. To see the reason for it, we resort to a simple special case with limited number of goods.

\subsection{Two privately-produced goods, one public}

Under this simple structure, and with $t_{1}=0, t_{2}$ and $\tau_{1}$ are found from equation (13) to be

$$
\left(\begin{array}{c}
t_{2} \\
\left(1+\frac{\delta}{\mu}\right) \tau_{1}
\end{array}\right)=\frac{-\delta}{\mu\left[\sum_{j} \pi^{j} \frac{\partial \tilde{x}_{2}^{j}}{\partial p_{2}} \sum_{j} \pi^{j} \frac{\partial \tilde{y}_{1}^{j}}{\partial q_{1}}-\left(\sum_{j} \pi^{j} \frac{\partial \tilde{x}_{2}^{j}}{\partial q_{1}}\right)^{2}\right]}\left(\begin{array}{c}
-\sum_{j} \pi^{j} \frac{\partial \tilde{x}_{2}^{j}}{\partial q_{1}} \sum_{j} \pi^{j} y_{1}^{j} \\
\sum_{j} \pi^{j} \frac{\partial \tilde{x}_{2}^{j}}{\partial p_{2}} \sum_{j} \pi^{j} y_{1}^{j}
\end{array}\right) .
$$

It immediately follows from the above that

$$
t_{2}=\frac{\sum_{j} \pi^{j} \frac{\partial \tilde{x}_{2}^{j}}{\partial q_{1}}}{-\sum_{j} \pi^{j} \frac{\partial \tilde{x}_{2}^{j}}{\partial p_{2}}}\left(1+\frac{\delta}{\mu}\right) \tau_{1} .
$$

With $\tau_{1}>0$ to cover the fixed costs, and $\partial \tilde{x}_{2}^{j} / \partial p_{2}<0, t_{2}$ has the same sign as $\sum_{j} \pi^{j}\left(\partial \tilde{x}_{2}^{j} / \partial q_{1}\right)=\sum_{j} \pi^{j}\left(\partial \tilde{y}_{1}^{j} / \partial p_{2}\right)$. Thus if $\partial \tilde{y}_{1}^{j} / \partial p_{2}>0$, one sets $t_{2}>0$. This increases $p_{2}$ and, with it, $\tilde{y}_{1}^{j}$. On the other hand, if $\partial \tilde{y}_{1}^{j} / \partial p_{2}<0$, one sets $t_{2}<0$. This lowers $p_{2}$ and, as a result, increases $\tilde{y}_{1}^{j}$. Either way then, one sets $t_{2}$ to increase $\tilde{y}_{1}$. 
The break-even condition increases the price of $y_{1}$ above its marginal cost so that its consumption is less than optimal. One attempts to reverse this through $t_{2}$. As a general lesson, taxation of privately-produced goods are necessitated to offset the distortions created by having to depart from marginal cost pricing on the part of the public firm.

The second important lesson we are seeking to answer is the extent to which the pricing rules defined by (13) resemble the traditional Ramsey rules. Traditionally, however, the Ramsey pricing rules are derived for either a unified government budget constraint (in the public finance literature), or for a public firm (in the regulation literature), but not for the two together as we have done here. Adding on a break-even constraint to the government's budget constraint in the Ramsey problem, however, does not change the structure of Ramsey taxes or Ramsey pricing rules. This is easy to establish. For completeness, and to establish a benchmark for comparison, we do this in the next section.

\section{Benchmark: the many-consumer Ramsey problem}

An individual of type $j$ now maximizes his utility $u=u(\underline{x}, \underline{y}, L)$ subject to the budget constraint $\sum_{i} p_{i} x_{i}+\sum_{s} q_{s} y_{s}=w^{j}(1-\theta) L+b$, where $\theta$ is the linear income tax rate and $b$ is the uniform lump-sum rebate. The resulting demand functions for $\underline{x}$ and $\underline{y}$, and the supply function for $L$, are denoted by $x_{i}^{j}=x_{i}\left(\underline{p}, \underline{q}, w^{j}(1-\theta), b\right), y_{s}^{j}=$ $y_{s}\left(\underline{p}, \underline{q}, w^{j}(1-\theta), b\right)$, and $L^{j}=L\left(\underline{p}, \underline{q}, w^{j}(1-\theta), b\right)$. Substituting in the utility function yields the indirect utility function

$$
\begin{aligned}
v^{j} & =v\left(\underline{p}, \underline{q}, w^{j}(1-\theta), b\right) \\
& \equiv u\left(\underline{x}\left(\underline{p}, \underline{q}, w^{j}(1-\theta), b\right), \underline{y}\left(\underline{p}, \underline{q}, w^{j}(1-\theta), b\right), L\left(\underline{p}, \underline{q}, w^{j}(1-\theta), b\right)\right) .
\end{aligned}
$$


Let $\pi^{j}$ denote the proportion of individuals of type $j$ in the economy and consider a utilitarian social welfare function of the form

$$
\sum_{j=1}^{H} \pi^{j} W\left(v^{j}\right),
$$

where $W(\cdot)$ is increasing, twice differentiable, and concave. The optimization problem consists of maximizing $\sum_{j} \pi^{j} W\left(v^{j}\right)$ with respect to $\underline{p}, \underline{q}, b$ and subject to resource and break-even constraints (8)-(9). Observe that with $\underline{x}$ and $\underline{y}$ being homogeneous of degree zero in $\underline{p}, \underline{q}, w^{j}(1-\theta)$ and $b$, consumer prices can determined only up to a proportionality factor. It is for this reason that we do not optimize over $\theta$, setting it equal to zero.

Define

$$
\begin{aligned}
\alpha^{j} \equiv & \frac{\partial v^{j}}{\partial b}, \\
\beta^{j} \equiv & \frac{1}{\mu} \frac{\partial W}{\partial v^{j}} \frac{\partial v^{j}}{\partial b}, \\
\gamma^{j} \equiv & \beta^{j}+\sum_{e=1}^{n} t_{e} \frac{\partial x_{e}^{j}}{\partial b}+\left(1+\frac{\delta}{\mu}\right) \sum_{f=1}^{m} \tau_{f} \frac{\partial y_{f}^{j}}{\partial b}, \\
\Omega \equiv & \left(\begin{array}{cccccc}
\sum_{j} \pi^{j} \frac{\partial \tilde{x}_{1}^{j}}{\partial p_{1}} & \cdots & \sum_{j} \pi^{j} \frac{\partial \tilde{x}_{1}^{j}}{\partial p_{n}} & \sum_{j} \pi^{j} \frac{\partial \tilde{x}_{1}^{j}}{\partial q_{1}} & \cdots & \sum_{j} \pi^{j} \frac{\partial \tilde{x}_{1}^{j}}{\partial q_{m}} \\
\vdots & \ddots & \vdots & \vdots & \ddots & \vdots \\
\sum_{j} \pi^{j} \frac{\partial_{n}^{j}}{\partial p_{1}} & \cdots & \sum_{j} \pi^{j} \frac{\partial \tilde{x}_{n}^{j}}{\partial p_{n}} & \sum_{j} \pi^{j} \frac{\partial \tilde{x}_{n}^{j}}{\partial q_{1}} & \cdots & \sum_{j} \pi^{j} \frac{\partial_{n}^{j}}{\partial q_{m}} \\
\sum_{j} \pi^{j} \frac{\partial \tilde{y}_{1}^{j}}{\partial p_{1}} & \cdots & \sum_{j} \pi^{j} \frac{\partial \tilde{y}_{1}^{j}}{\partial p_{n}} & \sum_{j} \pi^{j} \frac{\partial \tilde{y}_{1}^{j}}{\partial q_{1}} & \cdots & \sum_{j} \pi^{j} \frac{\partial \tilde{y}_{1}^{j}}{\partial q_{m}} \\
\vdots & \ddots & \vdots & \vdots & \ddots & \vdots \\
\sum_{j} \pi^{j} \frac{\partial \tilde{y}_{m}^{j}}{\partial p_{1}} & \cdots & \sum_{j} \pi^{j} \frac{\partial \tilde{y}_{m}^{j}}{\partial p_{n}} & \sum_{j} \pi^{j} \frac{\partial \tilde{y}_{m}^{j}}{\partial q_{1}} & \cdots & \sum_{j} \pi^{j} \frac{\partial \tilde{y}_{m}^{j}}{\partial q_{m}}
\end{array}\right) .
\end{aligned}
$$

Manipulating the first-order conditions of the government's optimization problem, given 
in the Appendix, we prove there,

$$
\left(\begin{array}{c}
t_{1} \\
\vdots \\
t_{n} \\
\left(1+\frac{\delta}{\mu}\right) \tau_{1} \\
\vdots \\
\left(1+\frac{\delta}{\mu}\right) \tau_{m}
\end{array}\right)=\Omega^{-1}\left(\begin{array}{c}
\sum_{j=1}^{H}\left(1-\gamma^{j}\right) \pi^{j} x_{1}^{j} \\
\vdots \\
\sum_{j=1}^{H}\left(1-\gamma^{j}\right) \pi^{j} x_{n}^{j} \\
\sum_{j=1}^{H}\left(1+\frac{\delta}{\mu}-\gamma^{j}\right) \pi^{j} y_{1}^{j} \\
\vdots \\
\sum_{j=1}^{H}\left(1+\frac{\delta}{\mu}-\gamma^{j}\right) \pi^{j} y_{m}^{j}
\end{array}\right)
$$

where we also prove that

$$
\sum_{j=1}^{H} \pi^{j} \gamma^{j}=1
$$

Observe that while the structure of taxes on private goods and the goods provided by the public firm are not identical, the same Ramsey tax/pricing rules apply to both under this setting. This is the benchmark with which one should compare and evaluate our result under nonlinear income taxes and weak separability presented in equation (13). ${ }^{11}$

The most striking difference between the sets of results is the lack of any distributional considerations in (13) as compared to (14) wherein distribution concerns enter through $\gamma^{j}$. This constitutes the second important lesson of our study: The tax/pricing rules for both types of goods, those that are produced privately and those that re provided through the public firm, are not affected by redistribution concerns.

To gain a better intuition into the nature of the tax/pricing rules, we next consider the two well-known special cases for which the Ramsey model yields simple results; namely, the cases of independent Hicksian and Marshallian demand curves. In both of these cases, the Ramsey solution implies the so called inverse elasticity rules. Following these cases, we examine the most celebrated general result of the Ramsey model; namely the (un)equal proportional reduction in compensated demands property.

\footnotetext{
${ }^{11}$ The many-consumer Ramsey problem is considered here while allowing for a uniform lump-sum rebate, $b$. The literature considers this problem for both cases when $b$ is and is not present. The same tax/pricing rules are derived in both cases. The only difference is that when $b$ is present, the optimization over $b$ results in $\sum_{j} \pi^{j} \gamma^{j}=1$. This result does not hold when $b$ is not present.
} 


\section{Pareto efficient versus Ramsey pricing rules}

\subsection{Zero cross-price compensated elasticities}

Assume that Hicksian demands are independent so that the compensated demand of any produced good does not depend on the prices of the other produced goods. In this case, the reduced Slutsky matrix (where the line and column pertaining to leisure is deleted) is diagonal so that equation (13) simplifies to

$$
\left(\begin{array}{c}
t_{2} \\
\vdots \\
t_{n} \\
\left(1+\frac{\delta}{\mu}\right) \tau_{1} \\
\vdots \\
\left(1+\frac{\delta}{\mu}\right) \tau_{m}
\end{array}\right)=\frac{\delta}{\mu}\left(\begin{array}{c}
0 \\
\vdots \\
0 \\
\frac{\sum_{j} \pi^{j} y_{1}^{j}}{-\sum_{j} \pi^{j} \frac{\partial \tilde{y}_{1}^{j}}{\partial q_{1}}} \\
\vdots \\
\frac{\sum_{j} \pi^{j} y_{m}^{j}}{-\sum_{j} \pi^{j} \frac{\partial \tilde{y}_{m}^{j}}{\partial q_{m}}}
\end{array}\right)
$$

Consequently, for all $i=1,2, \ldots, n$ and $s=1,2, \ldots, m$,

$$
\begin{aligned}
t_{i} & =0 \\
\tau_{s} & =\frac{\delta}{\mu+\delta} \frac{\sum_{j} \pi^{j} y_{s}^{j}}{-\sum_{j} \pi^{j} \frac{\partial \tilde{y}_{s}^{j}}{\partial q_{s}}}=\frac{\delta}{\mu+\delta} \frac{q_{s} \sum_{j} \pi^{j} y_{s}^{j}}{\sum_{j} \pi^{j} \tilde{y}_{s}^{j} \widetilde{\varepsilon}_{s s}^{j}},
\end{aligned}
$$

where $\widetilde{\varepsilon}_{s s}^{j}$ is the absolute value of the $j$-type's own-price elasticity of compensated demand for $y_{s}$. Or, for all $i=1,2, \ldots, n$ and $s=1,2, \ldots, m, t_{i}=0$ and

$$
\frac{\tau_{s}}{1+\tau_{s}}=\frac{\delta \sum_{j} \pi^{j} y_{s}^{j}}{(\mu+\delta) \sum_{j} \pi^{j} y_{s}^{j} \widetilde{\varepsilon}_{s s}^{j}},
$$

which is an inverse elasticity rule.

The above result is to be compared with the case of independent Hicksian demands under linear income taxes. There, the corresponding results are, for all $i=1,2, \ldots, n$ 
and $s=1,2, \ldots, m,{ }^{12}$

$$
\begin{aligned}
\frac{t_{i}}{1+t_{i}} & =\frac{\sum_{j}\left(1-\gamma^{j}\right) \pi^{j} x_{i}^{j}}{\sum_{j} \pi^{j} x_{i}^{j} \widetilde{\eta}_{i i}^{j}}=\frac{-\operatorname{Cov}\left(x_{i}^{j}, \gamma^{j}\right)}{\sum_{j} \pi^{j} x_{i}^{j} \widetilde{\eta}_{i i}^{j}}, \\
\frac{\tau_{s}}{1+\tau_{s}} & =\frac{\sum_{j}\left(1+\delta / \mu-\gamma^{j}\right) \pi^{j} y_{s}^{j}}{(1+\delta / \mu) \sum_{j} \pi^{j} y_{s}^{j} \widetilde{\varepsilon}_{s s}^{j}}=\frac{\delta \sum_{j} \pi^{j} y_{s}^{j}-\operatorname{Cov}\left(y_{s}^{j}, \gamma^{j}\right)}{(\mu+\delta) \sum_{j} \pi^{j} y_{s}^{j} \widetilde{\varepsilon}_{s s}^{j}},
\end{aligned}
$$

where $\widetilde{\eta}_{i i}^{j}$ is the absolute value of the $j$-type's own-price elasticity of compensated demands for $x_{i}$ and $\operatorname{Cov}(.,$.$) denotes covariance. Observe that in this traditional model,$ both types of goods, are subject to the inverse elasticity rules adjusted for redistribution benefits (through the covariance terms). However, with nonlinear income taxes and weak-separability, all covariance term disappear. This implies that there will be no tax on private goods while the goods provided by the public form are subject to the inverse elasticity rule that reflect pure efficiency considerations; see equation (15).

To sum, we find that in this special case, the private goods (not included in the break-even constraint) continue to go untaxed as in the AS setting. On the other hand, the pricing rules used by the public firm are purely efficiency-driven Ramsey rules. Goods are taxed inversely to their compensated demand elasticity regardless of their distributional implications. Redistribution is taken care of by the income tax allowing the public firm's prices to be adjusted for revenue raising (as in the Ramsey model with identical individuals).

It will become clear below that the apparent simplicity of this rule is to some extent misleading. It obscures some effects which are present but happen to cancel out in this special case. We shall return to this issue in the next subsections.

\footnotetext{
${ }^{12}$ These derivations are found by setting the cross-price derivatives in equations (A21)-(A22) equal to zero and rearranging the terms. The covariance interpretation follows because of the result that $\sum_{j} \gamma^{j}=1$.
} 


\subsection{Zero cross-price elasticities}

We now turn to the case where Marshallian demand functions are independent so that the demand for any given good does not depend on the prices of other (produced) goods. ${ }^{13}$ To simplify the pricing rules that obtain in this case, it is simpler to start from the intermediate expressions (A10)-(A11) given in the Appendix rather than from (13). Rearranging these expressions, making use of the weak-separability assumption, and setting all the cross-price derivatives equal to zero, we obtain for all $i=1,2, \ldots, n$ and $s=1,2, \ldots, m$,

$$
\begin{aligned}
& t_{i} \sum_{j} \pi^{j} \frac{\partial x_{i}^{j}}{\partial p_{i}}+\sum_{j} \pi^{j} x_{i}^{j}\left[\sum_{e=1}^{n} t_{e} \frac{\partial x_{e}^{j}}{\partial c^{j}}+\left(1+\frac{\delta}{\mu}\right) \sum_{f=1}^{m} \tau_{f} \frac{\partial y_{f}^{j}}{\partial c^{j}}\right]=0 \\
& \left(1+\frac{\delta}{\mu}\right) \tau_{s} \sum_{j} \pi^{j} \frac{\partial y_{s}^{j}}{\partial q_{s}}+\sum_{j} \pi^{j} y_{s}^{j}\left[\sum_{e=1}^{n} t_{e} \frac{\partial x_{e}^{j}}{\partial c^{j}}+\left(1+\frac{\delta}{\mu}\right) \sum_{f=1}^{m} \tau_{f} \frac{\partial y_{f}^{j}}{\partial c^{j}}\right]+\frac{\delta}{\mu} \sum_{j} \pi^{j} y_{s}^{j}=0 .
\end{aligned}
$$

Before simplifying these expressions any further, it is informative to delve into their interpretation. Recall that we are considering a compensated variation in the tax rates such that $d c_{j}=x_{i}^{j} d t_{i}$ for a variation in $t_{i}$ and $d c_{j}=y_{s}^{j} d q_{s}$ for a variation in $\tau_{s}$. In other words, individual disposable incomes are adjusted to keep utility levels constant for all individuals. With utility levels unchanged, the impact of the variation on social welfare entirely depends on the extra tax revenue (or profit) it generates. The left-hand sides of (18)-(19) measure the social value of this extra tax revenue (for a variation in $t_{i}$ or in $\tau_{s}$ respectively). Obviously, when the tax system is optimized, this social value must be equal to zero (otherwise welfare could be increased by changing the tax rates).

To understand this interpretation, assume one changes $c_{j}$ after $t_{i}$ or $\tau_{s}$ changes.

\footnotetext{
${ }^{13}$ Much of the regulation and IO literature uses quasi linear preference. In that case there are no income effects and the distinction between Hicksian and Marshallian demand becomes irrelevant.
} 
Start with a variation in $t_{i}$. With the tax revenues being given by

$$
\sum_{j} \pi^{j}\left(\sum_{e=1}^{n} t_{e} x_{e}^{j}+\sum_{f=1}^{m} \tau_{f} y_{f}^{j}\right)
$$

and the cross-price derivatives being equal to zero, the change in $t_{i}$ produces an extra tax revenue of

$$
\sum_{j} \pi^{j} x_{i}^{j}+t_{i} \sum_{j} \pi^{j} \frac{\partial x_{i}^{j}}{\partial p_{i}}
$$

Our compensation rule requires $\sum_{j} \pi^{j} x_{i}^{j}$ of this to be rebated to individuals. ${ }^{14}$ The net change in revenue, minus compensation, is equal to

$$
t_{i} \sum_{j} \pi^{j} \frac{\partial x_{i}^{j}}{\partial p_{i}}
$$

This is the first expression on the left-hand side of (18). At the same time, the $\sum_{j} \pi^{j} x_{i}^{j}$ compensation leads to an additional tax revenue of

$$
\sum_{e=1}^{n} t_{e} x_{e}^{j}\left(\sum_{j} \pi^{j} x_{i}^{j}\right)+\sum_{f=1}^{m} \tau_{f} y_{f}^{j}\left(\sum_{j} \pi^{j} x_{i}^{j}\right)=\left(\sum_{j} \pi^{j} x_{i}^{j}\right)\left(\sum_{e=1}^{n} t_{e} x_{e}^{j}+\sum_{f=1}^{m} \tau_{f} y_{f}^{j}\right) .
$$

To convert these tax revenue changes into social welfare (measured in units of general revenues), one must multiply tax revenue variations emanating from $\underline{y}$-goods by a factor of $(1+\delta / \mu)$. This is because the revenue from $\underline{y}$-goods enters both the global government budget constraint as well as the break-even constraint. This results in the second expression on the left-hand side of (18).

The left-hand side of (19) can be decomposed in a similar way, except for one extra complication; namely the additional term $(\delta / \mu) \sum_{j} \pi^{j} y_{s}^{j}$. In this exercise, $\sum_{j} \pi^{j} y_{s}^{j}$ represents the value of the refunds to individuals. When collected as a tax, this amount has a social value of $(1+\delta / \mu) \sum_{j} \pi^{j} y_{s}^{j}$. On the other hand, the refund "costs" only $\sum_{j} \pi^{j} y_{s}^{j}$ (it comes from the general budget and has no impact on the break even constraint).

\footnotetext{
${ }^{14}$ To see this, observe that $c_{j}$ changes according to $d c_{j}=x_{i}^{j} d t_{i}$ so that aggregate compensations change by $\sum_{j} \pi^{j} d c_{j}=\left(\sum_{j} \pi^{j} x_{i}^{j}\right) d t_{i}$.
} 
Finally, to ease the comparison with traditional Ramsey expressions, we can rewrite (18)-(19) as inverse elasticity rules. Introducing

$$
\begin{aligned}
& A_{i}=\sum_{j=1}^{H} \pi^{j} x_{i}^{j}\left[\sum_{e=1}^{n} t_{e} \frac{\partial x_{e}^{j}}{\partial c^{j}}+\left(1+\frac{\delta}{\mu}\right) \sum_{f=1}^{m} \tau_{f} \frac{\partial y_{f}^{j}}{\partial c^{j}}\right] \\
& B_{s}=\sum_{j=1}^{H} \pi^{j} y_{s}^{j}\left[\sum_{e=1}^{n} t_{e} \frac{\partial x_{e}^{j}}{\partial c^{j}}+\left(1+\frac{\delta}{\mu}\right) \sum_{f=1}^{m} \tau_{f} \frac{\partial y_{f}^{j}}{\partial c^{j}}\right]+\frac{\delta}{\mu} \sum_{j} \pi^{j} y_{s}^{j},
\end{aligned}
$$

where $A_{i}$ and $B_{s}$ measure the social value of the extra tax revenues due to refunds, with $B_{s}$ also including $(\delta / \mu) \sum_{j} \pi^{j} y_{s}^{j}$. We have, for all $i=1,2, \ldots, n$ and $s=1,2, \ldots, m$,

$$
\begin{aligned}
\frac{t_{i}}{1+t_{i}} & =\frac{A_{i}}{\sum_{j} \pi^{j} x_{i}^{j} \eta_{i i}^{j}}, \\
\frac{\tau_{s}}{1+\tau_{s}} & =\frac{B_{s}}{(1+\delta / \mu) \sum_{j} \pi^{j} y_{s}^{j} \varepsilon_{s s}^{j}},
\end{aligned}
$$

where and $\eta_{i i}^{j}$ and $\varepsilon_{s s}^{j}$ denote the absolute value of the $j$-type's own-price elasticity of Marshallian demands for $x_{i}$ and $y_{s}$.

Expressions (20)-(21) have a number of interesting implications, particularly when compared to their traditional counterparts. First, the effect of the break-even constraint is no longer confined to the goods which enter this constraint. Instead, it spills over to the other goods which no longer go untaxed; compare with the result obtained in Subsection 5.1. Second, we get inverse elasticity rules as in the Ramsey model; albeit without redistributive terms. This becomes clear below. The numerator of both expressions contain the "tax revenue" terms $A_{i}$ and $B_{s}$. Recall that these expressions measure the social value of the extra tax revenue generated from the demand variations that follow the (compensating) adjustments in disposable income.

One may wonder why these terms where absent in Subsection 5.1. The key to understanding this property is that when Hicksian demands are independent, the pricecum-income variations we consider have by definition no impact on the demand of any of the other goods. And the effect on the good under consideration is already captures in 
the (compensated) elasticity term. To sum up, Subsection 5.1 has given simple results, not because the different effects were absent but because they happen to cancel out exactly under the considered assumptions.

Finally, it is interesting to note that the counterparts of (20)-(21) in the traditional Ramsey model, under linear income taxes and independent Marshallian demands, are for all $i=1,2, \ldots, n$ and $s=1,2, \ldots, m$,

$$
\begin{aligned}
\frac{t_{i}}{1+t_{i}} & =\frac{A_{i}-\operatorname{Cov}\left(x_{i}^{j}, \gamma^{j}\right)}{\sum_{j} \pi^{j} x_{i}^{j} \eta_{i i}^{j}}, \\
\frac{\tau_{s}}{1+\tau_{s}} & =\frac{B_{s}-\operatorname{Cov}\left(y_{s}^{j}, \gamma^{j}\right)}{(1+\delta / \mu) \sum_{j} \pi^{j} y_{s}^{j} \varepsilon_{s s}^{j}} .
\end{aligned}
$$

These relationships are derived in the Appendix. Comparison of these relationships with (20)-(21) reveal that in our setup we have the exact expressions for optimal tax rates except for the covariance terms which vanish.

\subsection{Proportional reduction in compensated demands}

When there are cross-price effects, the Ramsey model no longer gives results that can be presented as "simply" as the inverse elasticity rules. One of the popular way to present the tax rules is in terms of proportional reduction in compensated demands. This leads to the celebrated "equal proportional reduction" results in the pure efficiency case, while the reduction depends on redistributive considerations in the many household case.

With separable preferences, one can rearrange equations (A12)-(A13) in the Appendix as follows

$$
\begin{aligned}
& -\mu \sum_{e=1}^{n} t_{e}\left(\sum_{j} \pi^{j} \frac{\partial \tilde{x}_{i}^{j}}{\partial p_{e}}\right)-\mu \sum_{f=1}^{m} \tau_{f}\left(\sum_{j} \pi^{j} \frac{\partial \tilde{x}_{i}^{j}}{\partial q_{f}}\right)=\delta \sum_{f=1}^{m} \tau_{f}\left(\sum_{j} \pi^{j} \frac{\partial \widetilde{y}_{f}^{j}}{\partial p_{i}}\right), \\
& -\mu \sum_{e=1}^{n} t_{e}\left(\sum_{j} \pi^{j} \frac{\partial \tilde{y}_{s}^{j}}{\partial p_{e}}\right)-\mu \sum_{f=1}^{m} \tau_{f}\left(\sum_{j} \pi^{j} \frac{\partial \tilde{y}_{s}^{j}}{\partial q_{f}}\right)=\delta \sum_{f=1}^{m} \tau_{f}\left(\sum_{j} \pi^{j} \frac{\partial \tilde{y}_{f}^{j}}{\partial q_{s}}\right)+\delta \sum_{j} \pi^{j} y_{s}^{j} .
\end{aligned}
$$


which can be written as

$$
\begin{aligned}
& -\frac{\sum_{e=1}^{n} t_{e}\left(\sum_{j} \pi^{j} \frac{\partial \tilde{x}_{i}^{j}}{\partial p_{e}}\right)+\sum_{f=1}^{m} \tau_{f}\left(\sum_{j} \pi^{j} \frac{\partial \tilde{x}_{i}^{j}}{\partial q_{f}}\right)}{\sum_{j} \pi^{j} x_{i}^{j}}=\frac{\delta}{\mu} \frac{\sum_{f=1}^{m} \tau_{f}\left(\sum_{j} \pi^{j} \frac{\partial \widetilde{y}_{f}^{j}}{\partial p_{i}}\right)}{\sum_{j} \pi^{j} x_{i}^{j}}, \\
& -\frac{\sum_{e=1}^{n} t_{e}\left(\sum_{j} \pi^{j} \frac{\partial \tilde{y}_{s}^{j}}{\partial p_{e}}\right)+\sum_{f=1}^{m} \tau_{f}\left(\sum_{j} \pi^{j} \frac{\partial \tilde{y}_{s}^{j}}{\partial q_{f}}\right)}{\sum_{j} \pi^{j} y_{s}^{j}}=\frac{\delta}{\mu}+\frac{\delta}{\mu} \frac{\sum_{f=1}^{m} \tau_{f}\left(\sum_{j} \pi^{j} \frac{\partial \tilde{y}_{f}^{j}}{\partial q_{s}}\right)}{\sum_{j} \pi^{j} y_{s}^{j}} \text {. }
\end{aligned}
$$

The left-hand side of (24) and (25) represents the proportional reduction in compensated demand of $x_{i}$ and $y_{i}$ respectively. Unlike in the single household Ramsey case it differs across goods. As a matter of fact, it is proportional to the compensated impact of the considered's goods tax rate on the break even constraint. Consequently we find that like in Subsection 5.2 redistributive considerations are replaced by tax revenue or more precisely revenue of the regulated firm.

These are to be compared with, for all $i=1,2, \ldots, n$ and $s=1,2, \ldots, m,{ }^{15}$

$$
\begin{gathered}
-\frac{\sum_{e=1}^{n} t_{e}\left(\sum_{j} \pi^{j} \frac{\partial \widetilde{x}_{i}^{j}}{\partial p_{e}}\right)+\sum_{f=1}^{m} \tau_{f}\left(\sum_{j} \pi^{j} \frac{\partial \widetilde{x}_{i}^{j}}{\partial q_{f}}\right)}{\sum_{j} \pi^{j} x_{i}^{j}}=\frac{\frac{\delta}{\mu} \sum_{f=1}^{m} \tau_{f}\left(\sum_{j} \pi^{j} \frac{\partial \widetilde{y}_{f}^{j}}{\partial p_{i}}\right)-\operatorname{Cov}\left(x_{i}^{j}, \gamma^{j}\right)}{\sum_{j} \pi^{j} x_{i}^{j}} \\
-\frac{\sum_{e=1}^{n} t_{e}\left(\sum_{j} \pi^{j} \frac{\partial \widetilde{y}_{s}^{j}}{\partial p_{e}}\right)+\sum_{f=1}^{m} \tau_{f}\left(\sum_{j} \pi^{j} \frac{\partial \widetilde{y}_{s}^{j}}{\partial q_{f}}\right)}{\sum_{j} \pi^{j} y_{s}^{j}}=\frac{\delta}{\mu}+\frac{\frac{\delta}{\mu} \sum_{f=1}^{m} \tau_{f}\left(\sum_{j} \pi^{j} \frac{\partial \tilde{y}_{f}^{j}}{\partial q_{s}}\right)-\operatorname{Cov}\left(y_{s}^{j}, \gamma^{j}\right)}{\sum_{j} \pi^{j} y_{s}^{j}} .
\end{gathered}
$$

One gain immediately observes that the two sets of formulas are identical except for the covariance terms which have finished in equations (24) and (25).

\footnotetext{
${ }^{15}$ This follows by rearranging equations (A21)-(A22) in the Appendix, where we have substituted $-\operatorname{Cov}\left(x_{i}^{j}, \gamma^{j}\right)$ for $\sum_{j}\left(1-\gamma^{j}\right) \pi^{j} x_{i}^{j}$ and $-\operatorname{Cov}\left(y_{s}^{j}, \gamma^{j}\right)$ for $\sum_{j}\left(1-\gamma^{j}\right) \pi^{j} y_{s}^{j}$.
} 


\section{Conclusion}

This paper has examined if the optimal tax and regulatory pricing approaches to Ramsey pricing can be reconciled. We have incorporate the two objectives of revenue raising for financing the government's expenditures (including redistributive transfers) and a regulated firm's fixed cost into a single framework. Except for the firm's break even constraint (which has no obvious informational justification) tax instruments were restricted only by informational considerations. Our setting is that of Atkinson and Stiglitz. More precisely, we have considered a mixed taxation setting à la Christiansen (1984) that combines nonlinear income taxation with linear pricing (taxation) of consumption goods.

The first major lesson that has emerged from our study is that the existence of a break-even constraint not only requires taxation of goods produced by the public/regulated firm but also the taxation of other goods. In other words, the breakeven constraints "spills over" to the other (non regulated) goods and affects their tax treatment.

To obtain more specific conclusions, we have considered two special cases for which the Ramsey model yields simple and well-known results; namely, the cases of independent Hicksian and Marshallian demand curves. In both the Ramsey solution implies the so-called inverse elasticity rule.

In the separable Hicksian demand case, the private goods (not included in the breakeven constraint) continue to go untaxed as in the Atkinson and Stiglitz setting. In the case where Marshallian demands are independent, the effect of the break-even constraint spills over to the other goods who no longer go untaxed. We continue to get inverse elasticity rules; however, their structure differs from the traditional expressions. In particular, there is no covariance (or similar) term that captures redistributive considerations. Instead, they contain "tax revenue terms" that measure the social value of the extra tax revenues generated from demand variations that follow the (compensating) 
adjustments in disposable income.

Finally, we revisit the most celebrated general result obtained in the Ramsey model; namely, the (un)equal proportional reduction in compensated demands property. We find that the redistributive considerations are once again replaced by tax revenue terms.

Our analysis could lead to a number of interesting extensions. First, it would be interesting to assess how significant the spill over effects on nonregulated goods are compared to the markups imposed on the goods included in the break-even constraint. Our various expressions suggest that this depends mainly on the significance of the (compensated) cross-price effects. However, the way in which they operate appears to be complex and not much can be said at this level of generality. While numerical examples could provide some illustrative indications a more satisfactory answer can only be provided by an empirical study. Second, our formulation relies on a traditional type of regulation which, while still applied in some sectors, has been given up or amended in many others. Consequently it would be interesting to extend our study to account for alternative regulatory arrangements, together with the introduction of competition and the use of more sophisticated pricing schemes in the relevant sectors. Last but not least, regulation very often pursues specific (often non welfarist) redistributive objective (like universal access) and it would be interesting to study how they interact with the general tax policy. 


\section{Appendix A}

First-order characterization of the(constrained) Pareto-efficient allocations:

Rearranging the terms in (11), and dropping the constants $\bar{R}$ and $F$, one may usefully rewrite the Lagrangian expression as

$$
\begin{aligned}
\mathcal{L}= & \sum_{j}\left(\eta^{j}+\sum_{k \neq j} \lambda^{j k}\right) v^{j}+\mu \sum_{j} \pi^{j}\left[\left(I^{j}-c^{j}\right)+\sum_{i=1}^{n}\left(p_{i}-1\right) x_{i}^{j}\right] \\
& +(\mu+\delta) \sum_{j} \pi^{j}\left[\sum_{s=1}^{m}\left(q_{s}-1\right) y_{s}^{j}\right]-\sum_{j} \sum_{k \neq j} \lambda^{j k} v^{j k} .
\end{aligned}
$$

The first-order conditions of this problem are, for $j, k=1,2, \ldots, H$, 


$$
\begin{aligned}
\frac{\partial \mathcal{L}}{\partial I^{j}} & =\left(\eta^{j}+\sum_{k \neq j} \lambda^{j k}\right) v_{I}^{j}+\mu \pi^{j}\left[1+\sum_{i=1}^{n}\left(p_{i}-1\right) \frac{\partial x_{i}^{j}}{\partial I^{j}}\right] \\
& +(\mu+\delta) \pi^{j}\left[\sum_{s=1}^{m}\left(q_{s}-1\right) \frac{\partial y_{s}^{j}}{\partial I^{j}}\right]-\sum_{k \neq j} \lambda^{k j} v_{I}^{k j}=0 \\
\frac{\partial \mathcal{L}}{\partial c^{j}} & =\left(\eta^{j}+\sum_{k \neq j} \lambda^{j k}\right) v_{c}^{j}+\mu \pi^{j}\left[-1+\sum_{i=1}^{n}\left(p_{i}-1\right) \frac{\partial x_{i}^{j}}{\partial c^{j}}\right] \\
& +(\mu+\delta) \pi^{j}\left[\sum_{s=1}^{m}\left(q_{s}-1\right) \frac{\partial y_{s}^{j}}{\partial c^{j}}\right]-\sum_{k \neq j} \lambda^{k j} v_{c}^{k j}=0, \\
\frac{\partial \mathcal{L}}{\partial p_{i}} & =\sum_{j}\left(\eta^{j}+\sum_{k \neq j} \lambda^{j k}\right) v_{i}^{j}+\mu \sum_{j} \pi^{j}\left[\sum_{e=1}^{n}\left(p_{e}-1\right) \frac{\partial x_{e}^{j}}{\partial p_{i}}+x_{i}^{j}\right] \\
& +(\mu+\delta) \sum_{j} \pi^{j}\left[\sum_{f=1}^{m}\left(q_{f}-1\right) \frac{\partial y_{f}^{j}}{\partial p_{i}}\right]-\sum_{j} \sum_{k \neq j} \lambda^{j k} v_{i}^{j k}=0, \quad i=2,3, \ldots, n, \quad(\mathrm{~A} 4) \\
\frac{\partial \mathcal{L}}{\partial q_{s}} & =\sum_{j}\left(\eta^{j}+\sum_{k \neq j} \lambda^{j k}\right) v_{s}^{j}+\mu \sum_{j} \pi^{j}\left[\sum_{e=1}^{n}\left(p_{e}-1\right) \frac{\partial x_{e}^{j}}{\partial q_{s}}\right] \\
& +(\mu+\delta) \sum_{j} \pi^{j}\left[\sum_{f=1}^{m}\left(q_{f}-1\right) \frac{\partial y_{f}^{j}}{\partial q_{s}}+y_{s}^{j}\right]-\sum_{j} \sum_{k \neq j} \lambda^{j k} v_{s}^{j k}=0, \quad s=1,2, \ldots, m,
\end{aligned}
$$

where the tax on $x_{1}^{j}$ is fixed at zero and a subscript on $v^{j}$ denotes a partial derivative. Equations (A2)-(A5) characterize the Pareto-efficient allocations constrained both by the public firms' break-even constraint, the resource constraint, the self-selection constraints, as well as the linearity of commodity tax rates.

Optimal commodity taxes: Multiply equation (A3) by $x_{i}^{j}$, sum over $j$ and add the resulting equation to (A4). Similarly, multiply (A3) by $y_{s}^{j}$, sum over $j$ and add the resulting equation to (A5). Simplifying results in the following system of equations for $i=2,3, \ldots, n$ and $s=1,2, \ldots, m$, 


$$
\begin{aligned}
& \frac{\partial \mathcal{L}}{\partial p_{i}}+\sum_{j} x_{i}^{j} \frac{\partial \mathcal{L}}{\partial c^{j}}= \\
& \sum_{j}\left(\eta^{j}+\sum_{k \neq j} \lambda^{j k}\right)\left(v_{i}^{j}+x_{i}^{j} v_{c}^{j}\right)+\mu \sum_{j} \pi^{j}\left[\sum_{e=1}^{n}\left(p_{e}-1\right)\left(\frac{\partial x_{e}^{j}}{\partial p_{i}}+x_{i}^{j} \frac{\partial x_{e}^{j}}{\partial c^{j}}\right)\right] \\
& +(\mu+\delta) \sum_{j} \pi^{j}\left[\sum_{f=1}^{m}\left(q_{f}-1\right)\left(\frac{\partial y_{f}^{j}}{\partial p_{i}}+x_{i}^{j} \frac{\partial y_{f}^{j}}{\partial c^{j}}\right)\right]-\sum_{j} \sum_{k \neq j} \lambda^{k j}\left(v_{i}^{k j}+x_{i}^{j} v_{c}^{k j}\right)=0, \\
& \frac{\partial \mathcal{L}}{\partial q_{s}}+\sum_{j} y_{s}^{j} \frac{\partial \mathcal{L}}{\partial c^{j}}= \\
& \sum_{j}\left(\eta^{j}+\sum_{k \neq j} \lambda^{j k}\right)\left(v_{s}^{j}+y_{s}^{j} v_{c}^{j}\right)+\mu \sum_{j} \pi^{j} \sum_{e=1}^{n}\left(p_{e}-1\right)\left(\frac{\partial x_{e}^{j}}{\partial q_{s}}+y_{s}^{j} \frac{\partial x_{e}^{j}}{\partial c^{j}}\right) \\
& +(\mu+\delta) \sum_{j} \pi^{j}\left[\sum_{f=1}^{m}\left(q_{f}-1\right)\left(\frac{\partial y_{f}^{j}}{\partial q_{s}}+y_{s}^{j} \frac{\partial y_{f}^{j}}{\partial c^{j}}\right)\right]-\sum_{j} \sum_{k \neq j} \lambda^{k j}\left(v_{s}^{k j}+y_{s}^{j} v_{c}^{k j}\right)+\delta \sum_{j} \pi^{j} y_{s}^{j}=0 .
\end{aligned}
$$

With $v_{i}^{j}+x_{i}^{j} v_{c}^{j}=0$ from Roy's identity, the left-hand side of (A6) shows the impact on the Lagrangian expression $\mathcal{L}$ of a variation in $p_{i}$ when the disposable income of individuals is adjusted according to

$$
d c_{j}=x_{i}^{j} d t_{i}
$$

to keep their utility levels constant. With $v_{s}^{j}+y_{s}^{j} v_{c}^{j}=0$, the left-hand side of (A7) shows the same compensated effect for a variation in $q_{s}$ where

$$
d c_{j}=y_{s}^{j} d \tau_{s}
$$

These compensated derivatives, $\left(\partial \mathcal{L} / \partial p_{i}\right)_{v^{j}=\bar{v}^{j}}$ and $\left(\partial \mathcal{L} / \partial q_{s}\right)_{v^{j}=\bar{v}^{j}}$ vanish at the optimal solution. 
Make use of Roy's identity to set,

$$
\begin{aligned}
v_{i}^{j}+x_{i}^{j} v_{c}^{j} & =0, \\
v_{i}^{k j}+x_{i}^{k j} v_{c}^{k j} & =0, \\
v_{s}^{j}+y_{s}^{j} v_{c}^{j} & =0, \\
v_{s}^{k j}+y_{s}^{k j} v_{c}^{k j} & =0 .
\end{aligned}
$$

Substitute these values in equations (A6)-(A7), set $p_{i}-1=t_{i}$ and $q_{s}-1=\tau_{s}$, and divide by $\mu$. Upon changing the order of summation and further simplification one arrives at, for all $i=2,3, \ldots, n$, and $s=1,2, \ldots, m$,

$$
\begin{aligned}
& \sum_{e=1}^{n} t_{e}\left[\sum_{j} \pi^{j}\left(\frac{\partial x_{e}^{j}}{\partial p_{i}}+x_{i}^{j} \frac{\partial x_{e}^{j}}{\partial c^{j}}\right)\right]+\left(1+\frac{\delta}{\mu}\right) \sum_{f=1}^{m} \tau_{f}\left[\sum_{j} \pi^{j}\left(\frac{\partial y_{f}^{j}}{\partial p_{i}}+x_{i}^{j} \frac{\partial y_{f}^{j}}{\partial c^{j}}\right)\right] \\
& -\frac{1}{\mu} \sum_{j} \sum_{k \neq j} \lambda^{k j}\left(x_{i}^{j}-x_{i}^{k j}\right) v_{c}^{k j}=0, \\
& \sum_{e=1}^{n} t_{e}\left[\sum_{j} \pi^{j}\left(\frac{\partial x_{e}^{j}}{\partial q_{s}}+y_{s}^{j} \frac{\partial x_{e}^{j}}{\partial c^{j}}\right)\right]+\left(1+\frac{\delta}{\mu}\right) \sum_{f=1}^{m} \tau_{f}\left[\sum_{j} \pi^{j}\left(\frac{\partial y_{f}^{j}}{\partial q_{s}}+y_{s}^{j} \frac{\partial y_{f}^{j}}{\partial c^{j}}\right)\right] \\
& -\frac{1}{\mu} \sum_{j} \sum_{k \neq j} \lambda^{k j}\left(y_{s}^{j}-y_{s}^{k j}\right) v_{c}^{k j}+\frac{\delta}{\mu} \sum_{j} \pi^{j} y_{s}^{j}=0 .
\end{aligned}
$$

Next, using the Slutsky equations,

$$
\begin{aligned}
\frac{\partial x_{e}^{j}}{\partial p_{i}} & =\frac{\partial \tilde{x}_{e}^{j}}{\partial p_{i}}-x_{i}^{j} \frac{\partial x_{e}^{j}}{\partial c^{j}} \\
\frac{\partial y_{f}^{j}}{\partial p_{i}} & =\frac{\partial \tilde{y}_{f}^{j}}{\partial p_{i}}-x_{i}^{j} \frac{\partial y_{f}^{j}}{\partial c^{j}} \\
\frac{\partial x_{e}^{j}}{\partial q_{s}} & =\frac{\partial \tilde{x}_{e}^{j}}{\partial q_{s}}-y_{s}^{j} \frac{\partial x_{e}^{j}}{\partial c^{j}}, \\
\frac{\partial y_{f}^{j}}{\partial q_{s}} & =\frac{\partial \tilde{y}_{f}^{j}}{\partial q_{s}}-y_{s}^{j} \frac{\partial y_{f}^{j}}{\partial c^{j}}
\end{aligned}
$$

while making use of the symmetry of the Slutsky matrix, one can further simplify 
(A10)-(A11) to

$$
\begin{aligned}
& \sum_{e=1}^{n} t_{e}\left(\sum_{j} \pi^{j} \frac{\partial \tilde{x}_{i}^{j}}{\partial p_{e}}\right)+\left(1+\frac{\delta}{\mu}\right) \sum_{f=1}^{m} \tau_{f}\left(\sum_{j} \pi^{j} \frac{\partial \tilde{x}_{i}^{j}}{\partial q_{f}}\right)=\frac{1}{\mu} \sum_{j} \sum_{k \neq j} \lambda^{k j}\left(x_{i}^{j}-x_{i}^{k j}\right) v_{c}^{k j} \\
& \sum_{e=1}^{n} t_{e}\left(\sum_{j} \pi^{j} \frac{\partial \tilde{y}_{s}^{j}}{\partial p_{e}}\right)+\left(1+\frac{\delta}{\mu}\right) \sum_{f=1}^{m} \tau_{f}\left(\sum_{j} \pi^{j} \frac{\partial \tilde{y}_{s}^{j}}{\partial q_{f}}\right)=\frac{1}{\mu} \sum_{j} \sum_{k \neq j} \lambda^{k j}\left(y_{s}^{j}-y_{s}^{k j}\right) v_{c}^{k j}-\frac{\delta}{\mu} \sum_{j} \pi^{j} y_{s}^{j},
\end{aligned}
$$

which hold for all $i=2,3, \ldots, n$, and $s=1,2, \ldots, m$. Finally, use the definition of $\Delta$ in (12) to write out equations (A12)-(A13) in matrix notation

$$
\Delta\left(\begin{array}{c}
t_{2} \\
\vdots \\
t_{n} \\
\left(1+\frac{\delta}{\mu}\right) \tau_{1} \\
\vdots \\
\left(1+\frac{\delta}{\mu}\right) \tau_{m}
\end{array}\right)=\frac{1}{\mu}\left(\begin{array}{c}
\sum_{j} \sum_{k \neq j} \lambda^{k j}\left(x_{2}^{j}-x_{2}^{k j}\right) v_{c}^{k j} \\
\vdots \\
\sum_{j} \sum_{k \neq j} \lambda^{k j}\left(x_{n}^{j}-x_{n}^{k j}\right) v_{c}^{k j} \\
\sum_{j} \sum_{k \neq j} \lambda^{k j}\left(y_{1}^{j}-y_{1}^{k j}\right) v_{c}^{k j} \\
\vdots \\
\sum_{j} \sum_{k \neq j} \lambda^{k j}\left(y_{m}^{j}-y_{m}^{k j}\right) v_{c}^{k j}
\end{array}\right)-\frac{\delta}{\mu}\left(\begin{array}{c}
0 \\
\vdots \\
0 \\
\sum_{j} \pi^{j} y_{1}^{j} \\
\vdots \\
\sum_{j} \pi^{j} y_{m}^{j}
\end{array}\right)
$$

Collecting the terms involving $\delta / \mu$ and premultiplying through by $\Delta^{-1}$ yields:

$$
\left(\begin{array}{c}
t_{2} \\
\vdots \\
t_{n} \\
\left(1+\frac{\delta}{\mu}\right) \tau_{1} \\
\vdots \\
\left(1+\frac{\delta}{\mu}\right) \tau_{m}
\end{array}\right)=\frac{1}{\mu} \Delta^{-1}\left(\begin{array}{c}
\sum_{j} \sum_{k \neq j} \lambda^{k j}\left(x_{2}^{j}-x_{2}^{k j}\right) v_{c}^{k j} \\
\vdots \\
\sum_{j} \sum_{k \neq j} \lambda^{k j}\left(x_{n}^{j}-x_{n}^{k j}\right) v_{c}^{k j} \\
\sum_{j} \sum_{k \neq j} \lambda^{k j}\left(y_{1}^{j}-y_{1}^{k j}\right) v_{c}^{k j} \\
\vdots \\
\sum_{j} \sum_{k \neq j} \lambda^{k j}\left(y_{m}^{j}-y_{m}^{k j}\right) v_{c}^{k j}
\end{array}\right)-\frac{\delta}{\mu} \Delta^{-1}\left(\begin{array}{c}
0 \\
\vdots \\
0 \\
\sum_{j} \pi^{j} y_{1}^{j} \\
\vdots \\
\sum_{j} \pi^{j} y_{m}^{j}
\end{array}\right) .
$$

Now, as shown in the text, weak-separability of preferences in labor supply and goods implies that $x_{i}^{j k}=x_{i}^{k}$ and $y_{s}^{j k}=y_{s}^{k}$ for all $i=1,2, \ldots, n$ and $s=1,2, \ldots, m$. 
This property reduces that the first matrix on the right-hand side of (A15) to zero so that (A15) simplifies to (13) as given in the text.

Derivation of (14), the Ramsey tax rule: The Lagrangian expression associated with maximizing $\sum_{j} \pi^{j} W\left(v^{j}\right)$ with respect to $\underline{p}, \underline{q}, b$ and subject to constraints (8)-(9) is represented by

$$
\begin{aligned}
\mathcal{L}= & \sum_{j} \pi^{j} W\left(v^{j}\right)+\mu\left\{\sum_{j} \pi^{j}\left[\sum_{i=1}^{n}\left(p_{i}-1\right) x_{i}^{j}+\sum_{s=1}^{m}\left(q_{s}-1\right) y_{s}^{j}-b\right]-\bar{R}-F\right\} \\
& +\delta\left\{\sum_{j} \pi^{j}\left[\sum_{s=1}^{m}\left(q_{s}-1\right) y_{s}^{j}\right]-F\right\} .
\end{aligned}
$$

The first-order conditions of this problem are,

$$
\begin{aligned}
\frac{\partial \mathcal{L}}{\partial b}=\sum_{j} \pi^{j} \frac{\partial W}{\partial v^{j}} \frac{\partial v^{j}}{\partial b}+\mu \sum_{j} \pi^{j}\left[-1+\sum_{i=1}^{n}\left(p_{i}-1\right) \frac{\partial x_{i}^{j}}{\partial b}\right]+ \\
(\mu+\delta) \sum_{j} \pi^{j}\left[\sum_{s=1}^{m}\left(q_{s}-1\right) \frac{\partial y_{s}^{j}}{\partial b}\right]=0, \\
\frac{\partial \mathcal{L}}{\partial p_{i}}=\sum_{j} \pi^{j} \frac{\partial W}{\partial v^{j}} \frac{\partial v^{j}}{\partial p_{i}}+\mu \sum_{j} \pi^{j}\left[x_{i}^{j}+\sum_{e=1}^{n}\left(p_{e}-1\right) \frac{\partial x_{e}^{j}}{\partial p_{i}}\right]+ \\
(\mu+\delta) \sum_{j} \pi^{j}\left[\sum_{f=1}^{m}\left(q_{f}-1\right) \frac{\partial y_{f}^{j}}{\partial p_{i}}\right]=0, \quad i=1,2, \ldots, n, \\
\frac{\partial \mathcal{L}}{\partial q_{s}}=\sum_{j} \pi^{j} \frac{\partial W}{\partial v^{j}} \frac{\partial v^{j}}{\partial q_{s}}+\mu \sum_{j} \pi^{j}\left[\sum_{e=1}^{n}\left(p_{e}-1\right) \frac{\partial x_{e}^{j}}{\partial q_{s}}\right]+ \\
(\mu+\delta) \sum_{j} \pi^{j}\left[y_{s}^{j}+\sum_{f=1}^{m}\left(q_{f}-1\right) \frac{\partial y_{f}^{j}}{\partial q_{s}}\right]=0, \quad s=1,2, \ldots, m .
\end{aligned}
$$

Using the definitions of $\alpha^{j}, \beta^{j}$, and $\gamma^{j}$ in the text, the first-order condition with respect to $b$, equation (A16), is simplified to 


$$
\sum_{j} \pi^{j} \gamma^{j}=1
$$

Next, using Roy's identity, the first-order conditions (A17)-(A18) are simplified to, for all $i=1,2, \ldots, n$, and $s=1,2, \ldots, m$,

$$
\begin{aligned}
& \sum_{j} \pi^{j}\left[-\beta^{j} x_{i}^{j}+\left(x_{i}^{j}+\sum_{e=1}^{n} t_{e} \frac{\partial x_{e}^{j}}{\partial p_{i}}\right)+\left(1+\frac{\delta}{\mu}\right) \sum_{f=1}^{m} \tau_{f} \frac{\partial y_{f}^{j}}{\partial p_{i}}\right]=0 \\
& \sum_{j} \pi^{j}\left[-\beta^{j} y_{s}^{j}+\left(\sum_{e=1}^{n} t_{e} \frac{\partial x_{e}^{j}}{\partial q_{s}}\right)+\left(1+\frac{\delta}{\mu}\right)\left(y_{s}^{j}+\sum_{f=1}^{m} \tau_{f} \frac{\partial y_{f}^{j}}{\partial q_{s}}\right)\right]=0 .
\end{aligned}
$$

Using the Slutsky equation and the symmetry of the Slutsky matrix, equations (A19)(A20) can also be written as

$$
\begin{aligned}
& -\left[\sum_{e=1}^{n} t_{e}\left(\sum_{j} \pi^{j} \frac{\partial \widetilde{x}_{i}^{j}}{\partial p_{e}}\right)+\left(1+\frac{\delta}{\mu}\right) \sum_{f=1}^{m} \tau_{f}\left(\sum_{j} \pi^{j} \frac{\partial \widetilde{x}_{i}^{j}}{\partial q_{f}}\right)\right]=\sum_{j} \pi^{j} x_{i}^{j}-\sum_{j} \gamma^{j} \pi^{j} x_{i}^{j}, \\
& -\left[\sum_{e=1}^{n} t_{e}\left(\sum_{j} \pi^{j} \frac{\partial \widetilde{y}_{s}^{j}}{\partial p_{e}}\right)+\left(1+\frac{\delta}{\mu}\right) \sum_{f=1}^{m} \tau_{f}\left(\sum_{j} \pi^{j} \frac{\partial \widetilde{y}_{s}^{j}}{\partial q_{f}}\right)\right]=\left(1+\frac{\delta}{\mu}\right) \sum_{j} \pi^{j} y_{s}^{j}-\sum_{j} \gamma^{j} \pi^{j} y_{s}^{j} .
\end{aligned}
$$

Finally, rewriting equations (A21)-(A22) in matrix notation, introducing and pre-multiplying by $\Omega^{-1}$ yields equation (14).

Derivation of (22)-(23): Set the cross-price derivatives in equations (A19)-(A20) equal to zero and rearrange the terms to get

$$
\begin{aligned}
\frac{t_{i}}{1+t_{i}} & =\frac{\sum_{j}\left(1-\beta^{j}\right) \pi^{j} x_{i}^{j}}{\sum_{j} \pi^{j} x_{i}^{j} \eta_{i i}^{j}} \\
\frac{\tau_{s}}{1+\tau_{s}} & =\frac{\sum_{j}\left(1+\delta / \mu-\beta^{j}\right) \pi^{j} y_{s}^{j}}{(1+\delta / \mu) \sum_{j} \pi^{j} y_{s}^{j} \varepsilon_{s s}^{j}} .
\end{aligned}
$$


Add and subtract $\gamma^{j}$ to $\beta^{j}$ to rewrite the above expressions as

$$
\begin{aligned}
\frac{t_{i}}{1+t_{i}} & =\frac{\sum_{j}\left(\gamma^{j}-\beta^{j}\right) \pi^{j} x_{i}^{j}-\sum_{j}\left(\gamma^{j}-1\right) \pi^{j} x_{i}^{j}}{\sum_{j} \pi^{j} x_{i}^{j} \eta_{i i}^{j}} \\
\frac{\tau_{s}}{1+\tau_{s}} & =\frac{\sum_{j}\left(\gamma^{j}-\beta^{j}\right) \pi^{j} y_{s}^{j}+(\delta / \mu) \sum_{j} \pi^{j} y_{s}^{j}-\sum_{j}\left(\gamma^{j}-1\right) \pi^{j} y_{s}^{j}}{(1+\delta / \mu) \sum_{j} \pi^{j} y_{s}^{j} \varepsilon_{s s}^{j}} .
\end{aligned}
$$

Rewrite $\sum_{j}\left(\gamma^{j}-1\right) \pi^{j} x_{i}^{j}$ and $\sum_{j}\left(\gamma^{j}-1\right) \pi^{j} y_{s}^{j}$ as covariances. Then recall from the definition of $\gamma^{j}$, that we have $\gamma^{j}-\beta^{j}=\sum_{e=1}^{n} t_{e}\left(\partial x_{e}^{j} / \partial b\right)+(1+\delta / \mu) \sum_{f=1}^{m} \tau_{f}\left(\partial y_{f}^{j} / \partial b\right)$. Substitute in above and make use of definition $A_{i}$ and $B_{s}$. 


\section{References}

[1] Atkinson, Anthony B. and Joseph E. Stiglitz, 1976. The design of tax structure: direct versus indirect taxation, Journal of Public Economics, 6, 55-75.

[2] Atkinson, Anthony B. and Joseph E. Stiglitz, 1980. Lectures on Public Economics, McGraw-Hill, New-York.

[3] Baumol, William J. and David F. Bradford, 1970. Optimal departures from marginal cost pricing, American Economic Review, 60, 265-283.

[4] Boiteux, Marcel, 1956. Sur la gestion des monopoles publics astreints à l'équilibre budgétaire, Econometrica, 24, 22-40. Published in English as: On the management of public monopolies subject to budgetary constraints, Journal of Economic Theory, 3, 219-240, 1971.

[5] Christiansen, Vidar, 1984. Which commodity taxes should supplement the income tax? Journal of Public Economics, 24, 195-220.

[6] Crew, Michael and Paul Kleindorfer, 2012. Non-linear pricing and volume discounts and the USO under entry, in Multi-modal Competition and the Future of Mail, M. A. Crew and P. R. Kleindorfer, ed., Northampton: Edward Elgar Publishing, Inc., $1-18$.

[7] Crew, Michael and Paul Kleindorfer, 2011. Competitive strategies under FMO and intermodal competition, in Reinventing the Postal Sector in an Electronic Age, M. A. Crew and P. R. Kleindorfer, ed., Northampton: Edward Elgar Publishing, Inc., $1-19$.

[8] Cremer, Helmuth and Firouz Gahvari, 1995. Uncertainty, optimal taxation and the direct versus indirect tax controversy, Economic Journal, 105, 1165-79.

[9] Cremer, Helmuth and Firouz Gahvari, 1998. On optimal taxation of housing", Journal of Urban Economics, 43, 315-335.

[10] Cremer, Helmuth and Firouz Gahvari, 2002. Nonlinear pricing, redistribution and optimal tax policy, Journal of Public Economic Theory, 4, 139-161. 
[11] Cremer, Helmuth, Firouz Gahvari and Norbert Ladoux, 1998. Externalities and optimal taxation, Journal of Public Economics, 70, 343-364.

[12] Cremer, Helmuth, Fahrid Gasmi, André Grimaux and Jean-Jacqaues Laffont, 2001. Universal Service: an economic perspective, Annals of Public and Cooperative Economics, 72, 5-43.

[13] Cremer, Helmuth, Pierre Pestieau and Jean-Charles Rochet, 2001. Direct versus indirect taxation : The design of the tax structure revisited, International Economic Review, 42, 781-799.

[14] Diamond, Peter A. and James A. Mirrlees, 1971. Optimal taxation and public production I-II, American Economic Review, 61, 8-27, 261-278.

[15] Diamond, Peter A., 1975. A many-person Ramsey tax rule, Journal of Public Economics, 4, 335-342.

[16] Laffont, Jean-Jacques and Jean Tirole, 1990. Bypass and cream skimming, American Economic Review, 80, 1042-1061.

[17] Laffont, Jean-Jacques and Jean Tirole, 2000. Competition in Telecommunications, MIT Press, Cambridge, Mass.

[18] Ramsey, Frank, 1927. A contribution to the theory of taxation, Economic Journal, 37, 47-61.

[19] Salanié, Bernard, 2003. The Economics of Taxation, MIT Press, Cambridge, Mass.

[20] Sandmo, Agnar, 1976. Optimal taxation: An introduction to the literature, Journal of Public Economics, 6, 37-54

[21] Vogelsang, Ingo et Jörg Finsinger, 1979. Regulatory adjustment process for optimal pricing of multiproduct firm, Bell Journal of Economics, 10, 157-171.

[22] Ware, Roger and Ralph A. Winter, 1986. Public pricing under imperfect competition, International Journal of Industrial Organization, 4, 87-97. 